\title{
ANALYSIS OF THE HETEROGENEOUS MULTISCALE METHOD FOR GAS DYNAMICS*
}

\author{
WEINAN $\mathrm{E}^{\dagger}$ AND XIANTAO $\mathrm{LI}^{\ddagger}$ \\ Dedicated to Professor George Papanicolaou on the occasion of his 60th birthday
}

\begin{abstract}
We carry out an error analysis for the heterogeneous multi-scale method for the case when the macroscale process is that of gas dynamics or more generally nonlinear conservation laws and the microscale process is an atomistic model such as kinetic Monte Carlo methods or molecular dynamics (MD). We will consider problems of type B as defined in [4], i.e. the macroscale constitutive relations are unknown and are extracted from the microscopic model. In addition to the standard error in the macroscale solver, a new error term occurs in estimating the data, here the fluxes. This new error term consists of three parts: the relaxation error, the sampling error and the error due to the finite size of the atomistic simulation. Our results serve as guidelines for designing multiscale methods, as was done in $[13,16]$.
\end{abstract}

Key words. multiscale methods, error estimates, conservation laws, molecular dynamics

AMS subject classifications. 65M15 35L65 70F10

1. Introduction. The main purpose of the present paper is to carry out an error analysis of the heterogeneous multiscale method for the case when the macroscale process is that of gas dynamics or more generally nonlinear conservation laws and the microscale process is an atomistic model such as kinetic Monte Carlo methods or molecular dynamics (MD). We will consider problems of type B as defined in [4], i.e. the macroscale constitutive relations are unknown and are extracted from the microscopic model.

An example of the microscopic model that we will consider is that of molecular dynamics, i.e. Newton's equations of motion for the constituting atoms,

$$
\left\{\begin{array}{c}
\dot{\mathbf{x}}_{i}=\mathbf{v}_{i}, \sum_{j \neq i} \mathbf{f}\left(\mathbf{x}_{i}(t)-\mathbf{x}_{j}(t)\right) . \\
m_{i} \dot{\mathbf{v}}_{i}
\end{array}\right.
$$

Here $m_{i}$ is the mass of the $i$-th particle and $\mathbf{f}(\mathbf{r})=-\nabla \phi(\mathbf{r})$ is the force exerted on the particles with interatomic potential $\phi(\mathbf{r})$.

The macroscale model is the continuum formulation of conservation laws of mass, momentum and energy, which are usually written in the following form,

$$
\left\{\begin{array}{c}
\partial_{t} \rho+\nabla_{\mathbf{x}} \cdot \mathbf{q}=0, \\
\partial_{t} \mathbf{q}+\nabla_{\mathbf{x}} \cdot \boldsymbol{\tau}=0, \\
\partial_{t} E+\nabla_{\mathbf{x}} \cdot \mathbf{J}=0 .
\end{array}\right.
$$

Here $\rho$ is the mass density of the system, $\mathbf{q}=\rho \mathbf{v}$ is the momentum density with $\mathbf{v}$ being the velocity, and $E$ is the total energy density. $\boldsymbol{\tau}$ and $\mathbf{J}$ are the momentum and energy fluxes respectively.

\footnotetext{
*Received October 26, 2004; accepted for publication December 3, 2004.

${ }^{\dagger}$ Department of Mathematics and PACM, Princeton University, Princeton, NJ 08544-1000, USA (weinan@math.princeton.edu).

${ }_{\ddagger}^{\ddagger}$ Institute for Mathematics and its Applications, University of Minnesota, Minneapolis, MN 554550436, USA (xli@ima.umn.edu.).
} 
Our point of departure from traditional continuum mechanics is in the treatment of constitutive relations. In continuum mechanics, it is customary to model the constitutive relations empirically. In contrast, we will obtain constitutive information from the underlying atomistic model. To see how this is done, observe that for MD (1.1), we can also write down the analogs of equations (1.2) by defining,

$$
\left\{\begin{aligned}
\widetilde{\rho}(\mathbf{x}, t) & =\sum_{i} m_{i} \delta\left(\mathbf{x}-\mathbf{x}_{i}(t)\right) \\
\widetilde{\mathbf{q}}(\mathbf{x}, t) & =\sum_{i} m_{i} \mathbf{v}_{i}(t) \delta\left(\mathbf{x}-\mathbf{x}_{i}(t)\right), \\
\widetilde{E}(\mathbf{x}, t) & =\frac{1}{2} \sum_{i}\left[m_{i} \mathbf{v}_{i}^{2}+\sum_{j \neq i} \phi\left(\mathbf{x}_{i}(t)-\mathbf{x}_{j}(t)\right)\right] \delta\left(\mathbf{x}-\mathbf{x}_{i}(t)\right),
\end{aligned}\right.
$$

and

$$
\begin{aligned}
\widetilde{\boldsymbol{\tau}}(\mathbf{x}, t) & =\sum_{i} m_{i} \mathbf{v}_{i} \otimes \mathbf{v}_{i} \delta\left(\mathbf{x}-\mathbf{x}_{i}(t)\right) \\
& +\frac{1}{2} \sum_{i} \sum_{j \neq i} \mathbf{f}\left(\mathbf{x}_{i}-\mathbf{x}_{j}\right) \otimes\left(\mathbf{x}_{i}-\mathbf{x}_{j}\right) \\
& \times \int_{0}^{1} \delta\left(\mathbf{x}-\left(\mathbf{x}_{j}+\lambda\left(\mathbf{x}_{i}-\mathbf{x}_{j}\right)\right)\right) d \lambda \\
\widetilde{\mathbf{J}}(\mathbf{x}, t) & =\sum_{i} \mathbf{v}_{i}\left[\frac{1}{2} m_{i} \mathbf{v}_{i}^{2}+\frac{1}{2} \sum_{j \neq i} \phi\left(\mathbf{x}_{i}(t)-\mathbf{x}_{j}(t)\right)\right] \delta\left(\mathbf{x}-\mathbf{x}_{i}(t)\right) \\
& +\frac{1}{4} \sum_{j \neq i}\left(\mathbf{v}_{j}(t)+\mathbf{v}_{i}(t)\right) \cdot \mathbf{f}\left(\mathbf{x}_{j}(t)-\mathbf{x}_{i}(t)\right)\left(\mathbf{x}_{i}-\mathbf{x}_{j}\right) \\
& \times \int_{0}^{1} \delta\left(\mathbf{x}-\left(\mathbf{x}_{j}+\lambda\left(\mathbf{x}_{i}-\mathbf{x}_{j}\right)\right)\right) d \lambda .
\end{aligned}
$$

One can easily verify that,

$$
\left\{\begin{array}{c}
\partial_{t} \widetilde{\rho}+\nabla_{\mathbf{x}} \cdot \widetilde{\mathbf{q}}=0, \\
\partial_{t} \widetilde{\mathbf{q}}+\nabla_{\mathbf{x}} \cdot \widetilde{\boldsymbol{\tau}}=0, \\
\partial_{t} \widetilde{E}+\nabla_{\mathbf{x}} \cdot \widetilde{\mathbf{J}}=0 .
\end{array}\right.
$$

The above calculation indicates that the continuum and atomistic models are consistent at the level of conservation laws. An additional observation is the scale separation: microscopic processes usually take place at the length scale of nanometers $\left(10^{-9} \mathrm{~m}\right)$ and time scale of femto-seconds $\left(10^{-15} \mathrm{~s}\right)$ on which one can neglect the variation of the macroscale variables. One can think of the atomistic system as been constrained by the macroscopic quantities, namely the mass, momentum and energy densities. In fact, the atomistic system will stay close to local equilibrium since there is sufficient time for relaxation to take place. One may thus view the models (1.2) as being the ensemble averages of (1.1).

Another example we will consider is the case when the microscopic model is the asymmetric simple exclusion process [11]. Consider the simplest case of a one dimensional lattice. Each lattice site is either unoccupied or occupied by a single particle. At Poission distributed times, a particle is picked at random, and with probability $p$ and $1-p(p \neq 1 / 2)$ respectively, the particle makes an attempted move 
to the nearest neighboring site to its left and its right. The move is accepted if the desired site is empty, and rejected otherwise.

In this case, it has been shown that the hydrodynamic equation, which plays the role of the macroscopic model for the present example, is the Burgers equation. Since for this problem, the passage from microscopic to macroscopic models is wellunderstood at the analytical level, it serves as a good example for the analysis of multi-scale, multi-physics numerical methods such as the heterogeneous multi-scale method.

2. The heterogeneous multi-scale method. The multi-scale method that we consider here has been developed in $[13,16]$, following the general framework of the heterogeneous multi-scale method (HMM), formulated in $[4,20]$. There are two main components in HMM: the selection of a macroscale solver and the estimation of the needed macroscale data using the microscale model. HMM has been applied to a wide range of physical problems. We refer to the review article [5] for a discussion of these applications as well as general issues of HMM. See also [20].

Macroscale solver. Our macroscopic model (1.2) is in the form of a conservation law, therefore we will choose as our macroscale solver a finite volume method. Although there are many different finite volume methods that are available for conservation laws e.g. $[12,10]$, many involve the computation of the Jacobian for the flux functions. These are not suitable for the present problem since the flux function is not explicitly given to us. An exception is the central scheme of Lax-Friedrichs type, such as [15], which is formulated over a staggered-grid. As it turns out, this method can be easily coupled with molecular dynamics.

To be more specific, we first rewrite the conservation laws in a generic form,

$$
\mathbf{u}_{t}+\mathbf{f}_{x}=0 .
$$

We will confine our discussion to macroscopically one dimensional problems since the extension to higher dimension is straightforward. The first order central scheme represents the solutions by piece-wise constants, which are the average values over each cell:

$$
\mathbf{u}_{k}^{n}=\frac{1}{\Delta x} \int_{x_{k-1 / 2}}^{x_{k+1 / 2}} \mathbf{u}\left(x, t^{n}\right) d x
$$

Time integration over $\left[x_{j}, x_{j+1}\right] \times\left[t^{n}, t^{n+1}\right)$ leads to the following scheme,

$$
\mathbf{u}_{k+1 / 2}^{n+1}=\frac{\mathbf{u}_{k}^{n}+\mathbf{u}_{k+1}^{n}}{2}-\frac{\Delta t}{\Delta x}\left(\mathbf{f}_{k+1}^{n}-\mathbf{f}_{k}^{n}\right),
$$

with numerical flux,

$$
\mathbf{f}_{k}^{n}=\frac{1}{\Delta t} \int_{t^{n}}^{t^{n+1}} \mathbf{f}\left(x_{k}, t\right) d t,
$$

which is taken to be $\mathbf{f}_{k}^{n}=\mathbf{f}\left(x_{k}, t^{n}\right)$ in central scheme.

(1.2) is still incomplete since we still do not know the fluxes. Next we describe how this information can be extracted from MD or other atomistic models.

Microscale solver. We first discuss the case when the microscopic model is MD. At each point where numerical fluxes are needed, we perform a local MD simulation to 
estimate the fluxes. The atomistic simulation will be constrained by the local macroscopic variables, which are the local density, momentum and energy. To initialize the MD simulation, we first arrange a trial configuration of the atoms according to the local density. From the local total energy, momentum and potential energy $V$ of the trial configuration, we can compute the thermal energy $K_{T}$ by

$$
K_{T}=E-V-\frac{1}{2} \rho \mathbf{v}^{2},
$$

If $K_{T}$ is negative, the trial configuration is rejected and another trial configuration with the same number of atoms is generated. Otherwise it is accepted, and a local initial temperature is estimated from,

$$
k_{B} T=\left\langle\frac{1}{d N} \sum_{i} m_{i} \mathbf{v}_{i}^{2}\right\rangle=\left\langle\frac{2}{d} K_{T}\right\rangle
$$

where $\langle\cdot\rangle$ is the ensemble average, $d$ is the dimension and $N$ is the number of particles in the MD system.

The velocities of the atoms are then given by

$$
\mathbf{v}_{i}=\mathbf{v}+\mathbf{v}_{i}^{\prime}
$$

where $\mathbf{v}_{i}^{\prime}$ is thermal velocity sampled from the Maxwell-Boltzmann distribution with the given local temperature. After the sampling, we adjust the instantaneous temperature to $T$ by rescaling $\mathbf{v}_{i}^{\prime}$ in order to satisfy (2.3).

The set of ODEs (1.1) can be solved by standard finite difference scheme such as Verlet's method. For the present analysis, however, we will assume that the ODEs are solved exactly to avoid unnecessary complication. After the MD system equilibrates, we obtain the needed quantities by averaging. Specifically let $\tilde{j}$ be the spatial average over the simulation box of a local flux whose expression was given in (1.4),

$$
\tilde{j}=\tilde{j}(\mathbf{X}), \quad \mathbf{X}=\left(\mathbf{x}_{1}, \mathbf{x}_{2}, \cdots, \mathbf{x}_{N}, \mathbf{v}_{1}, \mathbf{v}_{2}, \cdots, \mathbf{v}_{N}\right),
$$

then we obtain the corresponding macroscale quantity by time averaging,

$$
J=\frac{1}{T} \int_{\tau}^{\tau+T} \tilde{j}(\mathbf{X}(t)) d t
$$

where $\tau$ is the starting point when the time averaging begins, and $T$ is the duration of the time averaging. An additional ensemble averaging can also be used if desired. For detailed discussion on the setup of the MD as well as boundary conditions, see [13].

The overall numerical procedure is shown schematically in Figure 1. At each time step, the scheme (2.2) requires as input the fluxes at grid point $x_{k}$ to complete the time integration. These flux values are obtained by performing local MD simulations that are consistent with the local macroscale state $(\rho, \mathbf{q}, E)$. Once these values are computed, one can advance to the next macro time step using (2.2).

One result from such a method is shown in Figure 2. Here the setup for the macroscale model is a Riemann problem for one-dimensional wave propagation in solids. The result of HMM is compared with that of a direct MD simulation. The microscale model is two dimensional MD with Lennard-Jones potential.

If the microscopic model is the asymmetric simple exclusion process, the procedure is very similar. At each point where the numerical flux is needed, we set up a local 


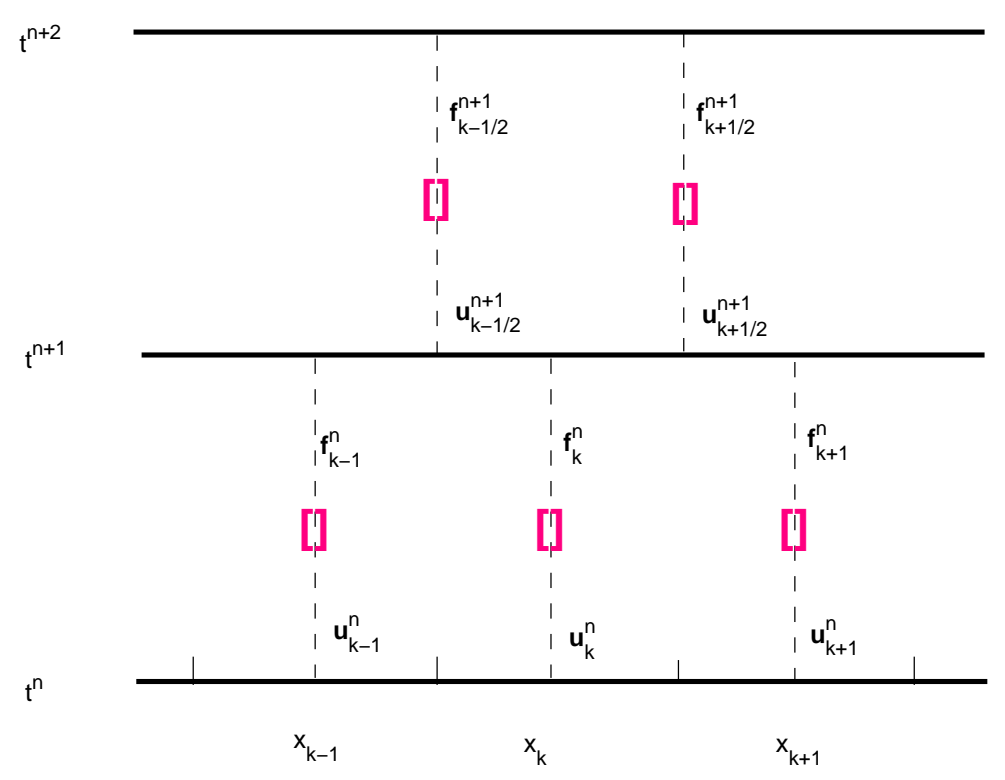

FIG. 1. A schematic illustration of the numerical procedure: starting from piece-wise constant solutions $\left\{\mathbf{u}_{k}^{n}\right\}$, one integrates (2.1) in time and in the cell $\left[x_{k}, x_{k+1}\right]$. The time step $\Delta t$ is chosen in such a way that the waves coming from $x_{k+1 / 2}$ will not reach $x_{k}$, and thus for $t \in\left[t^{n}, t^{n+1}\right), \mathbf{u}\left(x_{k}, t\right)=\mathbf{u}_{k}^{n}$. If $\mathbf{f}(\mathbf{u})$ at $x_{k}$ is found to be unknown, we perform a $M D$ simulation using $\mathbf{u}_{k}^{n}$ to invoke and restrict the microscopic process. The needed flux is then extracted from the simulation and the integration is completed. Analogously one can embed the MD simulation to higher order macro-schemes or higher dimensions.

simulation of the asymmetric exclusion process, with periodic boundary condition. Let $L$ be the total number of sites and $\rho_{j}^{n}$ be the current particle density at the $j$-th grid point. A total of $N=L \rho_{j}^{n}$ number of particles are distributed randomly according the simple exclusion rule. As the simulation proceeds, empirical fluxes are recorded at every micro time step, and the empirical flux is then averaged in time and ensemble, as discussed before for MD. This averaged value is then used in the macro solver to advance to the next macro time step.

3. Error analysis. The general strategy for the error analysis of such multiscale methods is as follows (see [4]). The first step is to analyze the error, $e(\mathrm{HMM})$ in estimating the macroscale data from the microscale model. The second step is to analyze the stability of the macroscale solver. If the macroscale solver is stable, then the total error in the overall scheme is the standard error of the macroscale solver plus an additional term due to the error in estimating the macroscale data. This strategy has been used for a number of examples including ODEs [3], stochastic ODEs [6] and homogenization problems [7, 14].

As the exact solution, when the microscale model is MD, we will take as the solution of the macroscale conservation law with fluxes given by averages of the microscopic fluxes with respect to the (local) infinite volume Gibbs state (see [17]). We will assume that the atomistic potential is smooth and bounded. When the microscale model is the simple asymmetric exclusion process, the exact solution is taken as the solution of the Burgers equation

$$
\rho_{t}+(p(1-p) \rho(1-\rho))_{x}=0
$$



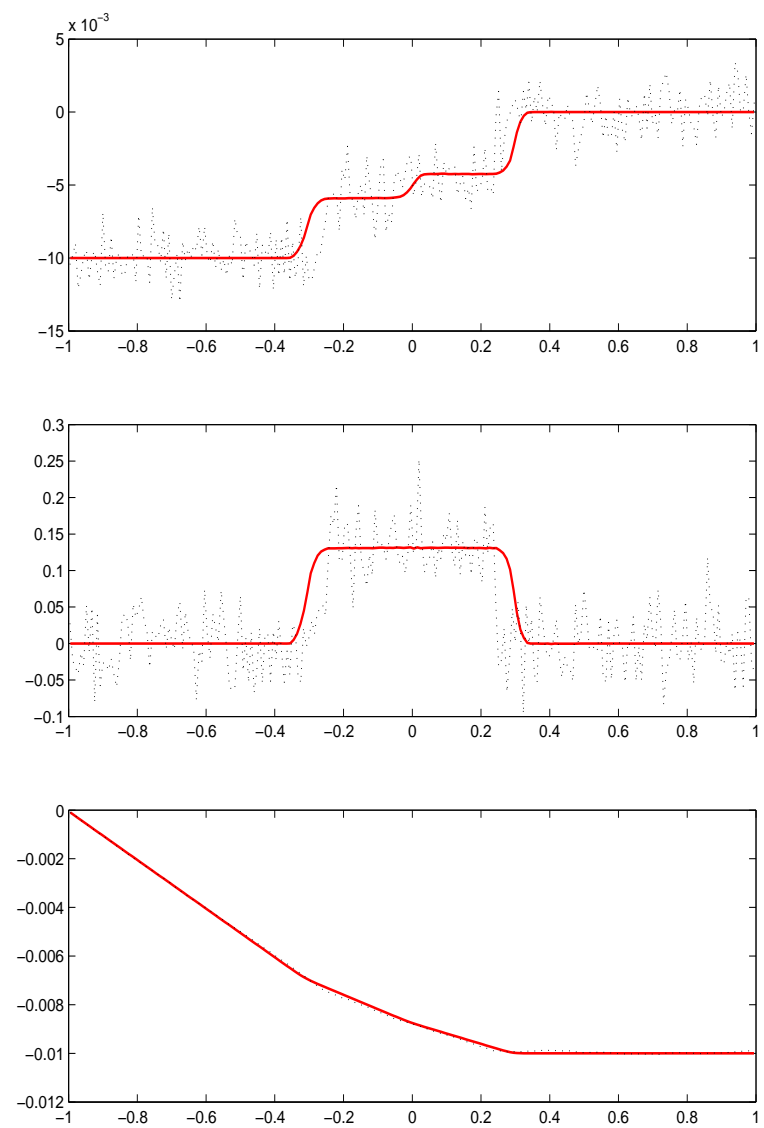

FIG. 2. Numerical test on shock formation and propagation. 200 macro-grid points are used and each local MD simulation consists of $40 \times 10$ atoms and $10^{4}$ steps of time integration. The solution is displayed after 40 steps of integration over macro time steps. Solid line: computed solution; dashed line: full atom simulation (one realization). Top: strain, middle: velocity, bottom: displacement.

that satisfies the entropy condition.

3.1. Error from estimating the fluxes. Let us first discuss the case when the microscale model is MD. Our goal now is to estimate the difference between the computed flux and the exact one:

$$
e(\mathrm{HMM})=E[|J-\bar{J}|],
$$

where the exact flux is defined to be the average w.r.t. the infinite volume Gibbs state $\mu_{\infty}$,

$$
\bar{J}=\int \tilde{j}(\mathbf{X}) d \mu_{\infty}
$$

and the computed flux $J$ is given by (2.6).

To proceed with the discussion, let us denote by $\mu_{L}^{t}$ the particle distribution for the MD system of size $L^{d}$ at time $t$ with $d$ being the dimension. We also define two 
quantities $\alpha(L)$ and $\tau_{0}(L)$ which are functions of $L$, the size of the microscale system, by the following

$$
\left|\int f(\mathbf{X}) d\left(\mu_{L}-\mu_{L}^{t}\right)\right| \leq C \mathrm{e}^{-\frac{t}{\tau_{0}(L)}}\|f\|_{w, \infty}
$$

and

$$
\left|\int f(\mathbf{X}) d\left(\mu_{L}-\mu_{\infty}\right)\right| \leq C \alpha(L)\|f\|_{w, \infty}
$$

for any continuous function $f$ that satisfies,

$$
\|f\|_{w, \infty} \leq \infty
$$

where,

$$
\|f\|_{w, \infty}=\sup _{\mathbf{X}}\left(1+|\mathbf{X}|^{2}\right)^{-p / 2}|f(\mathbf{X})|,
$$

for $p \geq 2$. $\tau_{0}(L)$ is the relaxation time for system of size $L$, and $\alpha(L)$ measures the error due to the finite size effect.

Now let

$$
J_{L}^{t}=\int \tilde{j}(\mathbf{X}) d \mu_{L}^{t}
$$

and the covariance,

$$
A(t, s)=E\left[\left(\tilde{j}(t)-J_{L}^{t}\right)\left(\tilde{j}(s)-J_{L}^{s}\right)\right],
$$

where the expectation is w.r.t. $\mu_{L}^{0}$, the initial distribution. We further assume that,

$$
\int_{0}^{+\infty} A(t, s) d s \leq C_{0}, \forall t \geq 0 .
$$

This amounts to assuming that the correlation decays in time sufficiently fast.

With $\left(A_{1}\right),\left(A_{2}\right)$, we have,

$$
\left|\bar{J}-\frac{1}{T} \int_{\tau}^{\tau+T} J_{L}^{t} d t\right| \leq C\left(\alpha(L)+\frac{1}{T} \mathrm{e}^{-\frac{t}{\tau_{0}(L)}}\right),
$$

and combining it with $\left(A_{3}\right)$, we have,

$$
\begin{aligned}
E\left[\left(J-\frac{1}{T} \int_{\tau}^{\tau+T} J_{L}^{t} d t\right)^{2}\right] & =\frac{1}{T^{2}} E\left[\int_{\tau}^{\tau+T} \int_{\tau}^{\tau+T}\left(\tilde{j}(t)-J_{L}^{t}\right)\left(\tilde{j}(s)-J_{L}^{s}\right) d t d s\right] \\
& \leq \frac{C_{0}}{T}
\end{aligned}
$$

In summary, we have

$$
e(\mathrm{HMM}) \leq C\left(\alpha(L)+\frac{1}{T} \mathrm{e}^{-\frac{\tau}{\tau_{0}(L)}}+\frac{1}{\sqrt{T}}\right) .
$$


If ensemble averaging is also used, for example, with $M$ independent copies, the above estimate becomes,

$$
e(\mathrm{HMM}) \leq C\left(\alpha(L)+\frac{1}{T} \mathrm{e}^{-\frac{\tau}{\tau_{0}(L)}}+\frac{1}{\sqrt{T M}}\right) .
$$

We clearly see that $e(\mathrm{HMM})$ is controlled by the behavior of $\alpha(L)$ and $\tau_{0}(L)$. Unfortunately, there are very few examples for which these two functions have been explicitly estimated. Almost all known examples are lattice systems, whereas MD is certainly an off-lattice systems.

In the absence of analytical results, we resort to careful numerical experiments to study how these terms behave for typical systems. Plotted in Figure 3 is a result on the finite size effect, expressed in $\alpha(L)$. In each $L$, we measure the stress component $\tau_{11}$ for sufficiently long time to eliminate the relaxation and sampling error. The exact value has been obtained from a simulation for large $L$. The error decays as the system size increases, consistent with the naive guess $L^{-1.5}$ from the central limit theorem.

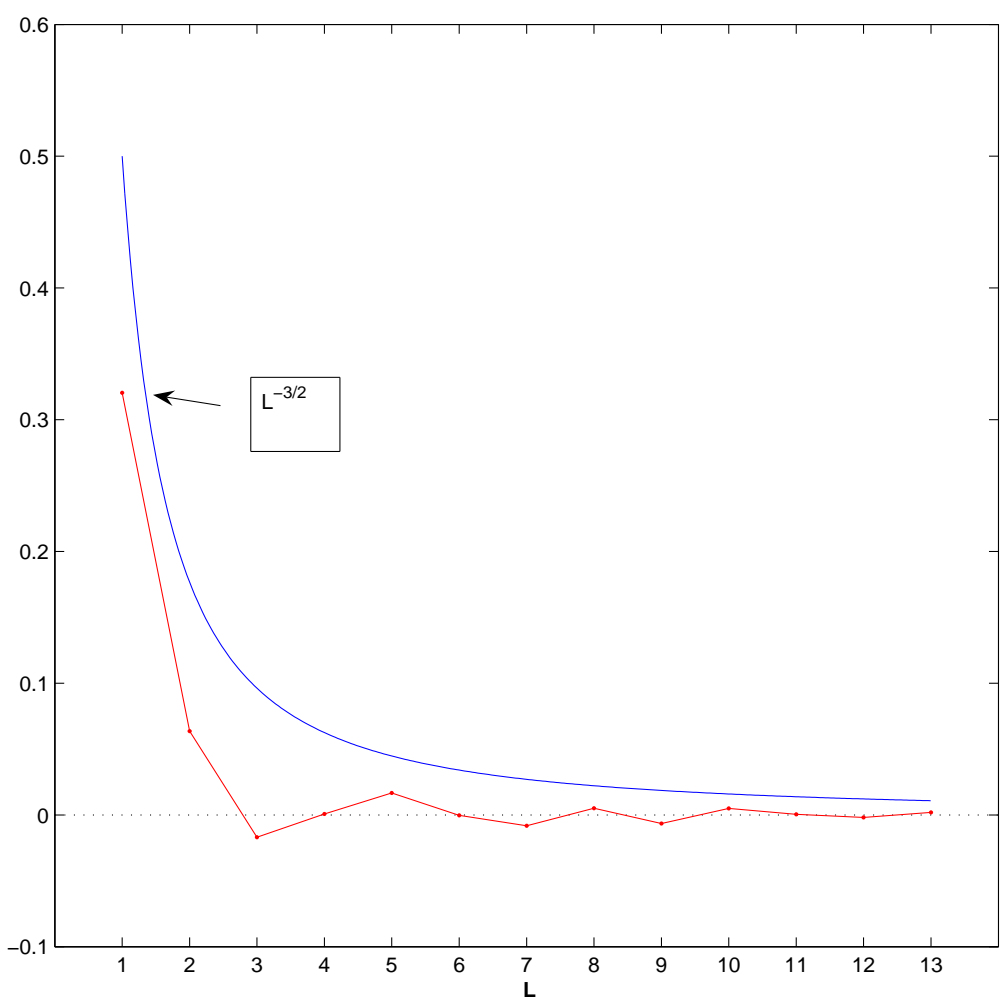

FIG. 3. Error due to finite size effect, plotted together with the $L^{-1.5}$.

The analysis proceeds in the same way for asymmetric simple exclusion process. However, in this case, the various quantities have been explicitly estimated. It is easy to see that

$$
\alpha(L)=0
$$

if $L \geq 3$, i.e. there are more than 3 sites in the micro cell. For the relaxation error, 
Lee and Yau [11] proved that,

$$
\tau_{0}(L) \leq C L^{2} \log L
$$

From (3.6), we see the conflicting effects of choosing the size of the MD system: when $L$ is increased, the finite size error is reduced while the relaxation time $\tau_{0}$ is increased. Similarly when $L$ is decreased, $\tau_{0}$ decreases but $\alpha(L)$ will increase.

3.2. Error analysis for the overall scheme. The macroscopic models considered in this paper have the following properties:

1. They are hyperbolic.

2. They have a strictly convex entropy function $\mathcal{S}$, with entropy flux $\mathbf{Q}$.

To avoid irrelevant complications, we will further assume that the numerical solutions at the macroscale level are bounded and the exact solution is piecewise Lipschitz continuous.

To begin with, we first define a new variable,

$$
\tilde{\mathbf{u}}=\nabla \mathcal{S}(\mathbf{u}) \text {. }
$$

Since the entropy is strictly convex, the mapping between $\tilde{\mathbf{u}}$ and $\mathbf{u}$ is one to one. We also introduce,

$$
\mathbf{H}=\frac{\partial \mathbf{u}}{\partial \tilde{\mathbf{u}}}, \quad \mathbf{B}=\nabla^{2} \mathcal{S}(\mathbf{u})
$$

and

$$
\psi(\tilde{\mathbf{u}})=\tilde{\mathbf{u}}^{\prime} \mathbf{f}(\mathbf{u}(\tilde{\mathbf{u}}))-\mathbf{Q}(\mathbf{u}(\tilde{\mathbf{u}}))
$$

with

$$
\frac{\partial \psi}{\partial \tilde{\mathbf{u}}}=\mathbf{f}(\mathbf{u}(\tilde{\mathbf{u}})) .
$$

Numerically (2.1) is often solved at first order by a three-point conservative scheme,

$$
\mathbf{u}_{j}^{n+1}=\mathbf{u}_{j}^{n}-\frac{\Delta t}{\Delta x}\left(\mathbf{f}_{j+1 / 2}^{n}-\mathbf{f}_{j-1 / 2}^{n}\right),
$$

where $\mathbf{f}_{j+1 / 2}=\mathbf{F}\left(\mathbf{u}_{j}, \mathbf{u}_{j+1}\right)$ and we have omitted the superscript. For Lax-Friedrichs type of schemes, which are the ones considered in the following analysis, the numerical flux $\mathbf{f}_{j+1 / 2}$ is given by,

$$
\mathbf{F}\left(\mathbf{u}_{j}, \mathbf{u}_{j+1}\right)=\frac{1}{2}\left(\mathbf{f}\left(\mathbf{u}_{j}\right)+\mathbf{f}\left(\mathbf{u}_{j+1}\right)\right)-\frac{a}{2}\left(\mathbf{u}_{j+1}-\mathbf{u}_{j}\right) .
$$

The constant $a$ is chosen to be bigger than the eigenvalues of $\nabla \mathbf{f}$

$$
a \geq \max |\lambda(\nabla \mathbf{f})|,
$$

to ensure stability. For the classical Lax-Friedrichs scheme, $a=\Delta x / \Delta t$ and for the two-step central scheme, $a=\Delta x /(2 \Delta t)$. Since the time step and the grid size are always proportional in the scheme, we will mainly use $\Delta x$ to indicate the discretization error. 
We need to analyze the effect on the numerical solution due to the error in the flux. Assume that we approximate the flux $f$ by $g$ :

$$
\begin{aligned}
\mathbf{u}_{j}^{n+1} & =\mathbf{u}_{j}^{n}-\frac{\Delta t}{\Delta x}\left(\mathbf{g}_{j+1 / 2}-\mathbf{g}_{j-1 / 2}\right), \\
\mathbf{g}_{j+1 / 2} & =\mathbf{G}\left(\mathbf{u}_{j}, \mathbf{u}_{j+1}\right),
\end{aligned}
$$

with,

$$
\mathbf{G}\left(\mathbf{u}_{j}, \mathbf{u}_{j+1}\right)=\frac{1}{2}\left(\mathbf{g}\left(\mathbf{u}_{j}\right)+\mathbf{g}\left(\mathbf{u}_{j+1}\right)\right)-\frac{a}{2}\left(\mathbf{u}_{j+1}-\mathbf{u}_{j}\right) .
$$

Let $\left\{\mathbf{v}_{j}^{n}\right\}$ be the numerical solution with exact flux function:

$$
\begin{aligned}
\mathbf{v}_{j}^{n+1} & =\mathbf{v}_{j}^{n}-\frac{\Delta t}{\Delta x}\left(\mathbf{f}_{j+1 / 2}-\mathbf{f}_{j-1 / 2}\right), \\
\mathbf{f}_{j+1 / 2} & =\mathbf{F}\left(\mathbf{v}_{j}, \mathbf{v}_{j+1}\right),
\end{aligned}
$$

and $\left\{\mathbf{w}_{j}^{n}\right\}$ be the intermediate variable:

$$
\begin{aligned}
\mathbf{w}_{j}^{n+1} & =\mathbf{u}_{j}^{n}-\frac{\Delta t}{\Delta x}\left(\mathbf{h}_{j+1 / 2}-\mathbf{h}_{j-1 / 2}\right), \\
\mathbf{h}_{j+1 / 2} & =\mathbf{F}\left(\mathbf{u}_{j}, \mathbf{u}_{j+1}\right) .
\end{aligned}
$$

Evidently within one time step, the difference between $\left\{\mathbf{w}_{j}^{n+1}\right\}$ and $\left\{\mathbf{u}_{j}^{n+1}\right\}$ can be controlled by $e(\mathrm{HMM})$,

$$
\left\|\mathbf{w}^{n+1}-\mathbf{u}^{n+1}\right\| \leq C e(\mathrm{HMM})
$$

with some constant $C$. The distance between $\left\{\mathbf{w}_{j}^{n+1}\right\}$ and $\left\{\mathbf{v}_{j}^{n}\right\}$, however, is directly related to the stability of the numerical methods.

For the scalar conservation laws, as in the case when the microscopic process is the asymmetric simple exclusion process, we have

THEOREM 1. Consider the case when the macroscale solver is the Lax-Friedrichs scheme. Fix $T>0$. Suppose that the CFL condition,

$$
a \frac{\Delta t}{\Delta x} \leq 1
$$

is satisfied, then

$$
\left\|u^{n}-v^{n}\right\|_{L_{1}} \leq C \frac{e(\mathrm{HMM})}{\Delta x}
$$

if $t=n \Delta t \leq T$. i.e.

Proof. From the $L_{1}$-contraction property of the Lax-Friedrichs scheme $[12,10]$,

$$
\left\|w^{n+1}-v^{n+1}\right\|_{L_{1}} \leq\left\|u^{n}-v^{n}\right\|_{L_{1}}
$$

and (3.17), one has,

$$
\left\|u^{n+1}-v^{n+1}\right\| \leq\left\|u^{n}-v^{n}\right\|_{L_{1}}+C e(\mathrm{HMM}) .
$$


Tracing back to $n=0$, one completes the proof.

For systems, we will make use of the functional introduced by DiPerna [2], to obtain the $L_{2}$ stability of the numerical solution. The result in [2] and a different version in [1] rely on the introduction of the functions,

$$
\begin{aligned}
\alpha(\mathbf{u}, \mathbf{v}) & =\mathcal{S}(\mathbf{u})-\mathcal{S}(\mathbf{v})-\nabla \mathcal{S}(\mathbf{v})(\mathbf{u}-\mathbf{v}) \\
\beta(\mathbf{u}, \mathbf{v}) & =\mathbf{Q}(\mathbf{u})-\mathbf{Q}(\mathbf{v})-\nabla \mathcal{S}(\mathbf{v})(\mathbf{f}(\mathbf{u})-\mathbf{f}(\mathbf{v}))
\end{aligned}
$$

First of all, $\alpha(\mathbf{u}, \mathbf{v})$ is a new entropy with entropy flux $\beta(\mathbf{u}, \mathbf{v})$ parameterized by $\mathbf{v}$, and $\alpha$ is the quadratic part of the entropy, and thus its integral is equivalent to the $L_{2}$ norm of $\mathbf{u}-\mathbf{v}$. Secondly, after some formal calculation, one finds,

$$
\alpha_{t}+\beta_{x}=-\mathbf{v}_{x}^{T} \nabla^{2} \mathcal{S}(\mathbf{v})(\mathbf{f}(\mathbf{u})-\mathbf{f}(\mathbf{v})-\nabla f(\mathbf{v})(\mathbf{u}-\mathbf{v})) .
$$

Therefore under the condition that $\mathbf{v}$ is a piece-wise Lipschitz solution, and $\mathbf{u}$ is a weak solution, one can show that

$$
\frac{d}{d t} \int \alpha(\mathbf{u}, \mathbf{v}) d x \leq C\|\mathbf{u}-\mathbf{v}\|_{L_{2}} .
$$

Hence the $L_{2}$ stability at the PDE level follows.

Nex we show the stability of semi-discrete version of (3.13):

Theorem 2. Assume that $\left\{\mathbf{v}_{j}\right\}$ and $\left\{\mathbf{u}_{j}\right\}$ are solutions of the semi-discrete scheme,

$$
\frac{d}{d t} \mathbf{u}_{j}=-\frac{1}{\Delta x}\left(\mathbf{F}\left(\mathbf{u}_{j}, \mathbf{u}_{j+1}\right)-\mathbf{F}\left(\mathbf{u}_{j-1}, \mathbf{u}_{j}\right)\right)
$$

and $\left\{\mathbf{v}_{j}\right\}$ satisfies

$$
\left\|\mathbf{v}_{j+1}^{n}-\mathbf{v}_{j}^{n}\right\|_{\infty} \leq C \Delta x, \quad\left\|\mathbf{v}_{j+1}^{n}-2 \mathbf{v}_{j}^{n}+\mathbf{v}_{j-1}^{n}\right\|_{\infty} \leq C \Delta x^{2},
$$

for all $j$ and $n \geq 0$. Then,

$$
\sum_{j} \alpha\left(\mathbf{u}_{j}^{n+1}, \mathbf{v}_{j}^{n+1}\right) \Delta x \leq(1+C \Delta t) \sum_{j} \alpha\left(\mathbf{u}_{j}^{n}, \mathbf{v}_{j}^{n}\right) \Delta x+C \Delta x^{2} .
$$

Here $C$ denotes generic constants.

Proof. We first prove the entropy inequality for the numerical scheme. Detailed discussion on the entropy stability can be found in the review paper by Tadmor [19]. First recall that $\tilde{\mathbf{u}}=\nabla \mathcal{S}(\mathbf{u})$. By direct calculation one has,

$$
\begin{aligned}
\frac{d}{d t} \mathcal{S}\left(\mathbf{u}_{j}\right) & =-\frac{1}{\Delta x}\left(\mathbf{Q}\left(\mathbf{u}_{j}, \mathbf{u}_{j+1}\right)-\mathbf{Q}\left(\mathbf{u}_{j-1}, \mathbf{u}_{j}\right)\right) \\
& +\frac{1}{2 \Delta x}\left(\left(\mathbf{u}_{j+1}-\tilde{\mathbf{u}}_{j}\right)^{T} \mathbf{f}_{j+1 / 2}-\left(\psi\left(\tilde{\mathbf{u}}_{j+1}\right)-\psi\left(\tilde{\mathbf{u}}_{j}\right)\right)\right) \\
& +\frac{1}{2 \Delta x}\left(\left(\tilde{\mathbf{u}}_{j}-\tilde{\mathbf{u}}_{j-1}\right)^{T} \mathbf{f}_{j-1 / 2}-\left(\psi\left(\tilde{\mathbf{u}}_{j}\right)-\psi\left(\tilde{\mathbf{u}}_{j-1}\right)\right)\right) .
\end{aligned}
$$

Now taking $\tilde{\mathbf{u}}_{j+1 / 2}(\xi)=\frac{1}{2}\left(\tilde{\mathbf{u}}_{j}+\tilde{\mathbf{u}}_{j+1}\right)+\xi\left(\tilde{\mathbf{u}}_{j+1}-\tilde{\mathbf{u}}_{j}\right)$, one gets:

$$
\begin{aligned}
\psi\left(\tilde{\mathbf{u}}_{j+1}\right)-\psi\left(\tilde{\mathbf{u}}_{j}\right) & =\left(\tilde{\mathbf{u}}_{j+1}-\tilde{\mathbf{u}}_{j}\right)^{T} \int_{-1 / 2}^{1 / 2} \mathbf{f}\left(\mathbf{u}\left(\tilde{\mathbf{u}}_{j+1 / 2}(\xi)\right)\right) d \xi \\
& =\frac{1}{2}\left(\tilde{\mathbf{u}}_{j+1}-\tilde{\mathbf{u}}_{j}\right)^{T}\left(\mathbf{f}\left(\mathbf{u}_{j}\right)+\mathbf{f}\left(\mathbf{u}_{j+1}\right)\right) \\
& -\left(\tilde{\mathbf{u}}_{j+1}-\tilde{\mathbf{u}}_{j}\right)^{T} \int_{-1 / 2}^{1 / 2} \xi \nabla \mathbf{f H} d \xi\left(\tilde{\mathbf{u}}_{j+1}-\tilde{\mathbf{u}}_{j}\right),
\end{aligned}
$$


and,

$$
\begin{aligned}
\left(\tilde{\mathbf{u}}_{j+1}-\tilde{\mathbf{u}}_{j}\right)^{T} \mathbf{f}_{j+1 / 2} & =\frac{1}{2}\left(\tilde{\mathbf{u}}_{j+1}-\tilde{\mathbf{u}}_{j}\right)^{T}\left(\mathbf{f}\left(\mathbf{u}_{j}\right)+\mathbf{f}\left(\mathbf{u}_{j+1}\right)\right) \\
& -\frac{a}{2}\left(\tilde{\mathbf{u}}_{j+1}-\tilde{\mathbf{u}}_{j}\right)^{T}\left(\mathbf{u}_{j+1}-\mathbf{u}_{j}\right) \\
& =\frac{1}{2}\left(\tilde{\mathbf{u}}_{j+1}-\tilde{\mathbf{u}}_{j}\right)^{T}\left(\mathbf{f}\left(\mathbf{u}_{j}\right)+\mathbf{f}\left(\mathbf{u}_{j+1}\right)\right) \\
& -\frac{a}{2}\left(\tilde{\mathbf{u}}_{j+1}-\tilde{\mathbf{u}}_{j}\right)^{T} \int_{-1 / 2}^{1 / 2} \mathbf{H} d \xi\left(\tilde{\mathbf{u}}_{j+1}-\tilde{\mathbf{u}}_{j}\right) .
\end{aligned}
$$

Since $\frac{\partial \mathbf{v}}{\partial \mathbf{u}}=\nabla^{2} \mathcal{S}$ is positive definite, $\nabla \mathbf{f H}=\mathbf{H}^{-1 / 2}\left(\mathbf{H}^{1 / 2} \nabla \mathbf{f} \mathbf{H}^{1 / 2}\right) \mathbf{H}^{1 / 2}$ and $a \geq$ $\max |\lambda(\nabla \mathbf{f})|$, we have

$$
\frac{d}{d t} \mathcal{S}\left(\mathbf{u}_{j}\right) \leq-\frac{1}{\Delta x}\left(\mathbf{Q}\left(\mathbf{u}_{j}, \mathbf{u}_{j+1}\right)-\mathbf{Q}\left(\mathbf{u}_{j-1}, \mathbf{u}_{j}\right)\right) .
$$

For the smooth solution $\mathbf{v}$, the inequality becomes equality within order of $\Delta x$ :

$$
\frac{d}{d t} \mathcal{S}\left(\mathbf{v}_{j}\right)=-\frac{1}{\Delta x}\left(\mathbf{Q}\left(\mathbf{v}_{j}, \mathbf{v}_{j+1}\right)-\mathbf{Q}\left(\mathbf{v}_{j-1}, \mathbf{v}_{j}\right)\right)+O(\Delta x)
$$

Now we compute the time derivative of $\alpha(\mathbf{u}, \mathbf{v})$. First notice that,

$$
\begin{aligned}
& \nabla \mathcal{S}\left(\mathbf{v}_{j}\right) \frac{d}{d t}\left(\mathbf{u}_{j}-\mathbf{v}_{j}\right) \\
= & \left.-\tilde{\mathbf{v}}_{j}^{T}\left(\mathbf{F}\left(\mathbf{u}_{j}, \mathbf{u}_{j+1}\right)-\mathbf{F}\left(\mathbf{u}_{j-1}, \mathbf{u}_{j}\right)-\mathbf{F}\left(\mathbf{v}_{j}, \mathbf{v}_{j+1}\right)\right)+\mathbf{F}\left(\mathbf{v}_{j-1}, \mathbf{v}_{j}\right)\right) / \Delta x \\
= & -\tilde{\mathbf{v}}_{j+1 / 2}^{T}\left(\mathbf{F}\left(\mathbf{u}_{j}, \mathbf{u}_{j+1}\right)-\mathbf{F}\left(\mathbf{v}_{j}, \mathbf{v}_{j+1}\right)\right) / \Delta x \\
+ & \tilde{\mathbf{v}}_{j-1 / 2}^{T}\left(\mathbf{F}\left(\mathbf{u}_{j-1}, \mathbf{u}_{j}\right)-\mathbf{F}\left(\mathbf{v}_{j-1}, \mathbf{v}_{j}\right)\right) / \Delta x \\
+ & \frac{1}{2}\left(\tilde{\mathbf{v}}_{j+1}^{T}-\tilde{\mathbf{v}}_{j}^{T}\right)\left(\mathbf{F}\left(\mathbf{u}_{j}, \mathbf{u}_{j+1}\right)-\mathbf{F}\left(\mathbf{v}_{j}, \mathbf{v}_{j+1}\right)\right) / \Delta x \\
+ & \frac{1}{2}\left(\tilde{\mathbf{v}}_{j}^{T}-\tilde{\mathbf{v}}_{j-1}^{T}\right)\left(\mathbf{F}\left(\mathbf{u}_{j-1}, \mathbf{u}_{j}\right)-\mathbf{F}\left(\mathbf{v}_{j-1}, \mathbf{v}_{j}\right)\right) / \Delta x .
\end{aligned}
$$

By splitting $\tilde{\mathbf{v}}_{j}^{T}$ we obtain two conservative terms, which can be eliminated by summation. One can continue to extract conservative terms as follows,

$$
\begin{aligned}
& \frac{1}{2}\left(\tilde{\mathbf{v}}_{j+1}^{T}-\tilde{\mathbf{v}}_{j}^{T}\right)\left(\mathbf{F}\left(\mathbf{u}_{j}, \mathbf{u}_{j+1}\right)-\mathbf{F}\left(\mathbf{v}_{j}, \mathbf{v}_{j+1}\right)\right) / \Delta x \\
= & \frac{1}{4}\left(\tilde{\mathbf{v}}_{j+1}^{T}-\tilde{\mathbf{v}}_{j}^{T}\right)\left(\mathbf{f}\left(\mathbf{u}_{j+1}\right)-\mathbf{f}\left(\mathbf{u}_{j}\right)+\mathbf{f}\left(\mathbf{v}_{j+1}\right)-\mathbf{f}\left(\mathbf{v}_{j}\right)\right) / \Delta x \\
+ & \frac{a}{4}\left(\tilde{\mathbf{v}}_{j+1}^{T}-\tilde{\mathbf{v}}_{j}^{T}\right)\left(\mathbf{u}_{j+1}+\mathbf{u}_{j}-\mathbf{v}_{j+1}+\mathbf{v}_{j}\right) / \Delta x \\
+ & \frac{1}{2}\left(\tilde{\mathbf{v}}_{j+1}-\tilde{\mathbf{v}}_{j}\right)^{T}\left(\mathbf{f}\left(\mathbf{u}_{j}\right)-\mathbf{f}\left(\mathbf{v}_{j}\right)\right) / \Delta x \\
+ & \frac{a}{2}\left(\mathbf{u}_{j}-\mathbf{v}_{j}\right)^{T}\left(\tilde{\mathbf{v}}_{j+1}-\tilde{\mathbf{v}}_{j}\right) / \Delta x .
\end{aligned}
$$


With the entropy inequalities and the above calculation, one has,

$$
\begin{aligned}
& \frac{d}{d t} \alpha\left(\mathbf{u}_{j}, \mathbf{v}_{j}\right) \\
\leq & -\frac{1}{\Delta x}\left(\mathbf{Q}\left(\mathbf{u}_{j}, \mathbf{u}_{j+1}\right)-\mathbf{Q}\left(\mathbf{u}_{j-1}, \mathbf{u}_{j}\right)\right)+\frac{1}{\Delta x}\left(\mathbf{Q}\left(\mathbf{v}_{j}, \mathbf{v}_{j+1}\right)-\mathbf{Q}\left(\mathbf{v}_{j-1}, \mathbf{v}_{j}\right)\right) \\
+ & \tilde{\mathbf{v}}_{j+1 / 2}^{T}\left(\mathbf{F}\left(\mathbf{u}_{j}, \mathbf{u}_{j+1}\right)-\mathbf{F}\left(\mathbf{v}_{j}, \mathbf{v}_{j+1}\right)\right) / \Delta x \\
- & \tilde{\mathbf{v}}_{j-1 / 2}^{T}\left(\mathbf{F}\left(\mathbf{u}_{j-1}, \mathbf{u}_{j}\right)-\mathbf{F}\left(\mathbf{v}_{j-1}, \mathbf{v}_{j}\right)\right) / \Delta x \\
- & \frac{1}{4}\left(\left(\tilde{\mathbf{v}}_{j+1}-\tilde{\mathbf{v}}_{j}\right)^{T}\left(\mathbf{f}\left(\mathbf{u}_{j+1}\right)-\mathbf{f}\left(\mathbf{u}_{j}\right)\right)-\left(\tilde{\mathbf{v}}_{j}-\tilde{\mathbf{v}}_{j-1}\right)^{T}\left(\mathbf{f}\left(\mathbf{u}_{j}\right)-\mathbf{f}\left(\mathbf{u}_{j-1}\right)\right)\right) / \Delta x \\
+ & \frac{1}{4}\left(\left(\tilde{\mathbf{v}}_{j+1}-\tilde{\mathbf{v}}_{j}\right)^{T}\left(\mathbf{f}\left(\mathbf{v}_{j+1}\right)-\mathbf{f}\left(\mathbf{v}_{j}\right)\right)-\left(\tilde{\mathbf{v}}_{j}-\tilde{\mathbf{v}}_{j-1}\right)^{T}\left(\mathbf{f}\left(\mathbf{v}_{j}\right)-\mathbf{f}\left(\mathbf{v}_{j-1}\right)\right)\right) / \Delta x \\
- & \frac{1}{2}\left(\tilde{\mathbf{v}}_{j+1}-\tilde{\mathbf{v}}_{j-1}\right)^{T}\left(\mathbf{f}\left(\mathbf{u}_{j}\right)-\mathbf{f}\left(\mathbf{v}_{j}\right)\right) / \Delta x \\
+ & \frac{a}{4}\left(\left(\tilde{\mathbf{v}}_{j+1}-\tilde{\mathbf{v}}_{j}\right)^{T}\left(\mathbf{u}_{j+1}+\mathbf{u}_{j}\right)-\left(\tilde{\mathbf{v}}_{j}-\tilde{\mathbf{v}}_{j-1}\right)^{T}\left(\mathbf{u}_{j}+\mathbf{u}_{j-1}\right)\right) / \Delta x \\
- & \frac{a}{4}\left(\left(\tilde{\mathbf{v}}_{j+1}-\tilde{\mathbf{v}}_{j}^{T}\right)\left(\mathbf{v}_{j+1}+\mathbf{v}_{j}\right)-\left(\tilde{\mathbf{v}}_{j}-\tilde{\mathbf{v}}_{j-1}\right)^{T}\left(\mathbf{v}_{j}+\mathbf{v}_{j-1}\right)\right) / \Delta x \\
- & \frac{a}{2}\left(\mathbf{u}_{j}-\mathbf{v}_{j}\right)^{T}\left(\tilde{\mathbf{v}}_{j+1}-2 \tilde{\mathbf{v}}_{j}+\tilde{\mathbf{v}}_{j-1}\right) / \Delta x \\
+ & \frac{1}{2}\left(\mathbf{v}_{j+1}-\mathbf{v}_{j-1}\right)^{T} \nabla^{2} \mathcal{S} \nabla \mathbf{f}\left(\mathbf{v}_{j}\right)\left(\mathbf{u}_{j}-\mathbf{v}_{j}\right) / \Delta x+O(\Delta x) \\
= & -\frac{1}{2}\left(\tilde{\mathbf{v}}_{j+1}-\tilde{\mathbf{v}}_{j}\right)^{T} \nabla^{2} \mathcal{S}\left(\mathbf{v}_{j}\right)\left(\mathbf{f}\left(\mathbf{u}_{j}\right)-\mathbf{f}\left(\mathbf{v}_{j}\right)-\nabla \mathbf{f}\left(\mathbf{v}_{j}\right)\left(\mathbf{u}_{j}-\mathbf{v}_{j}\right)\right) / \Delta x \\
+ & O(\Delta x)+\operatorname{conservative~terms.~}
\end{aligned}
$$

This implies that,

$$
\frac{d}{d t} \sum_{j} \alpha\left(\mathbf{u}_{j}, \mathbf{v}_{j}\right) \Delta x \leq C \sum_{j} \alpha\left(\mathbf{u}_{j}, \mathbf{v}_{j}\right) \Delta x+O(\Delta x),
$$

and (3.22) follows immediately. $\mathrm{Q}$

In light of (3.17) and the entropy inequality (3.23), we obtain the entropy stability of our numerical solution $\left\{\mathbf{u}_{j}^{n}\right\}$. An additional fact we will use is that In addition suppose that the the Lax-Friedrichs scheme is first order accurate for smooth solutions in the $L_{2}$ norm. Together, we then obtain

Theorem 3. Let $\mathbf{u}$ be the exact solution to the PDEs (2.1), and $\mathbf{u}$ is smooth. Let $\mathbf{u}_{\mathrm{HMM}}=\left\{\mathbf{u}_{j}^{n}\right\}$, we have

$$
\sum_{j} \mathcal{S}\left(\mathbf{u}_{j}^{n}\right) \Delta x \leq \sum_{j} \mathcal{S}\left(\mathbf{u}_{j}^{0}\right) \Delta x+C \Delta x+C \frac{e(\mathrm{HMM})}{\Delta x}
$$

and

$$
\left\|\mathbf{u}-\mathbf{u}_{\mathrm{HMM}}\right\|_{L_{2}} \leq C \Delta x+\sqrt{C \Delta x+C \frac{e(\mathrm{HMM})}{\Delta x}} .
$$

Proof. First notice that (3.10) and (3.13) are the Euler discretization of the semi-discrete schemes. In discrete form, we have the entropy inequality,

$$
\sum_{j} \mathcal{S}\left(\mathbf{w}_{j}^{n+1}\right) \Delta x \leq \sum_{j} \mathcal{S}\left(\mathbf{u}_{j}^{n}\right) \Delta x+C \Delta x^{2} .
$$


In light of (3.17), one gets,

$$
\sum_{j} \mathcal{S}\left(\mathbf{u}_{j}^{n+1}\right) \Delta x \leq \sum_{j} \mathcal{S}\left(\mathbf{u}_{j}^{n}\right) \Delta x+C \Delta x^{2}+C e(\mathrm{HMM}),
$$

which then gives (3.25).

Similarly from the estimate of the functional $\alpha$, we have

$$
\sum_{j} \alpha\left(\mathbf{w}_{j}^{n+1}, \mathbf{v}_{j}^{n+1}\right) \Delta x \leq(1+C \Delta t) \sum_{j} \alpha\left(\mathbf{u}_{j}^{n}, \mathbf{v}_{j}^{n}\right) \Delta x+C \Delta x^{2},
$$

and,

$$
\sum_{j} \alpha\left(\mathbf{u}_{j}^{n+1}, \mathbf{v}_{j}^{n+1}\right) \Delta x \leq(1+C \Delta t) \sum_{j} \alpha\left(\mathbf{u}_{j}^{n}, \mathbf{v}_{j}^{n}\right) \Delta x+C \Delta x^{2}+C e(\mathrm{HMM}) .
$$

Thus we conclude that (3.26) holds.

To see whether this estimate is sharp, we have conducted a number of numerical experiments in which the size of the macro grid is changed systematically, with all parameters fixed. The 'exact' equation of state is obtained from a series of large scale MD simulations (in multiscale modeling, this is referred to as the serial coupling technique), and the exact solution is computed on a very fine grid using the exact equation of state. In Fig. 4, we plot the total error versus the grid size. We see that for large $\Delta x$, the error scales as $\Delta x$. This behavior changes to $\Delta x^{1 / 2}$ for smaller values of $\Delta x$. But we do not observe the $\Delta x^{-1 / 2}$ type of behavior suggested by the analytical result.

This discrepancy might be explained as follows. In the analysis presented above, we did not take into account the possible cancellations between the $e(\mathrm{HMM})$ terms at neighboring grid points. This is because $e(\mathrm{HMM})$ contains atomistic fluctuations which are essentially independent for the different microscopic simulations at different grid points, therefore we do not expect to see cancellations. In the case when the microscopic simulation is a Monte Carlo scheme, this actually suggests a possible technique for variance reduction, by using the same set of random numbers on different grid points. Nevertheless, in general, one does not expect cancellation from the fluctuation part of $e(\mathrm{HMM})$.

However, this does not exclude the possibility that the fluctuation part of $e(\mathrm{HMM})$ is very small, compared with the other parts which are smooth, and a better estimate can be obtained by taking into account the cancellations in the remaining part of $e(\mathrm{HMM})$. This is what is suggested in Figure 4.

In addition, the relaxation error can be reduced by using a better initialization scheme for the microscopic solver. For ODEs, this is discussed in detail in [5].

We remark that the results can be easily extended to multi-dimensional case. Indeed the stability of the solutions for hyperbolic systems was indeed originally established for systems in arbitrary dimensions, and the entropy stability of the Lax-Friedrichs scheme in higher dimension is also straightforward. The estimation of $e(\mathrm{HMM})$ does not rely on dimension, even though the specific dependence of $\alpha(L)$ and $\tau_{0}(L)$ may very well do depend on dimension.

Acknowledgement. It is a pleasure to thank Bjorn Engquist, Weiqing Ren and Eitan Tadmor for stimulating discussions regarding this work. This work is supported in part by an ONR grant N00014-01-1-0674. 


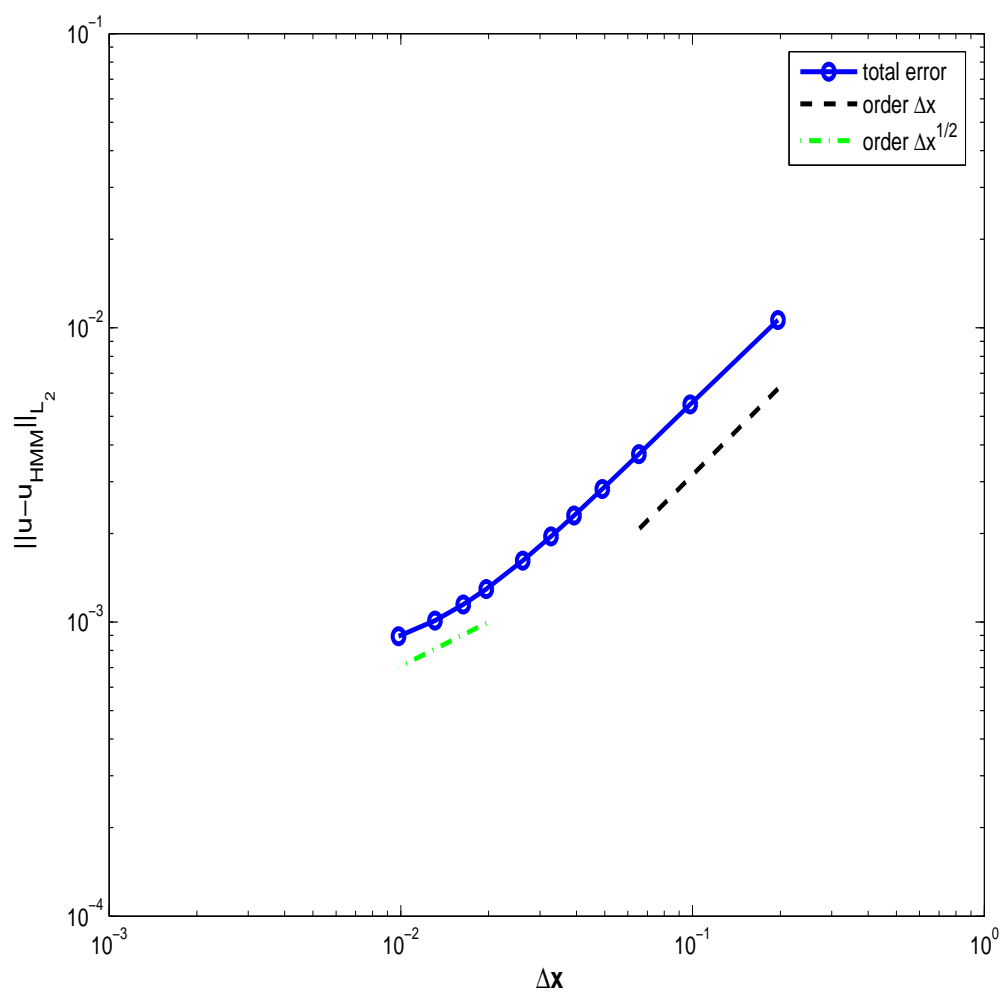

FIG. 4. Dependence of the total error on the grid size. The data is plotted at log-log scale.

\section{REFERENCES}

[1] C.M. Dafermos, Hyperbolic conservation laws in continuum physics, Springer-Verlag, Berlin, 2000.

[2] R.J. DiPerna, Uniqueness of solutions to hyperbolic conservation laws, Ind. Univ. Math. J., 28 (1979), pp. 27-70.

[3] W. E, Analysis of the heterogeneous multiscale method for ordinary differential equations, Comm. Math. Sci., 1:3 (2003), pp. 423-436.

[4] W. E and B. Engquist, The Heterogeneous multi-scale methods, Comm. Math. Sci., 1:1 (2003), pp. $87-132$.

[5] W. E, B. Engquist, X. Li, W. Ren and E. Vanden-Eijnden, The Heterogeneous Multiscale Method: A Review, Lecture Notes in Mathematics, to appear.

[6] W. E, D. Liu And E. Vanden-Eijnden, Analysis of numerical techniques for multiscale stochastic dynamical systems, Comm. Pure Appl. Math., to appear.

[7] W. E, P. B. Ming And P. W. Zhang, Analysis of the heterogeneous multiscale method for elliptic homogenization problems, J. Amer. Math. Soc., 18 (2005), pp. 121-156.

[8] R.S. ELLIS, Entropy, large deviations, and statistical mechanics, Springer-Verlag, New York, 1985.

[9] H. Flyvbjerg and H.G. Petersen, Error estimates on averages of correlated data, J. Chem. Phys., 91:1 (1989), pp. 461-466.

[10] E. Godlewsini And P.A. Raviart, Numerical Approximation of hyperbolic systems of conservation laws, Springer-Verlag, New York, 1996.

[11] T.Y. Lee And H.T. YAU, Logarithmic Sobolev inequality for some models of random walks, Ann. Probab., 26:4 (1998), pp. 1855-1873.

[12] R. LeVeque, Numerical methods for conservation laws, Birkhäuser, 1992.

[13] X.T. LI AND W. E, Multiscale modeling for dynamics of solids at finite temperature, J. Mech. Phys. solids, 53 (2004), pp. 1650-1685. 
[14] P. B. Ming And P. W. Zhang, Analysis of the heterogeneous multiscale method for parabolic homogenization problems, submitted to Math. Comp.

[15] H. Nessyahu AND E. TADMOR, Nonoscillatory central differencing for hyperbolic conservation laws, J. Comp. Phys., 87:2 (1990), pp. 408-463.

[16] W. Ren And W. E, Heterogeneous multiscale method for the modeling of complex fluids and microfluidics, J. Comp. Phys., 204 (2005), pp. 1-26.

[17] H. Spohn, Large Scale Dynamics of Interacting Particles, Springer-Verlag, 1991.

[18] G. Strang, Accurate partial difference methods. II. Non-linear problems, Numer. Math., 6 (1964), pp. 37-46.

[19] E. TADMOR, Entropy stability theory for difference approximations of nonlinear conservation laws and related time-dependent problems, Acta Numerica, (2003), pp. 451-512.

[20] Visit http://www.pacm.princeton.edu/multiscale for applications of HMM and recent progress. 